International Journal of Pure and Applied Mathematics

Volume 85 No. 3 2013, 593-607

ISSN: 1311-8080 (printed version); ISSN: 1314-3395 (on-line version)

url: http://www.ijpam.eu

doi: http://dx.doi.org/10.12732/ijpam.v85i3.14

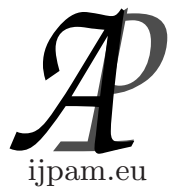

\title{
ON RELATIVE GI-INJECTIVE, GF-PROJECTIVE AND GI-FLAT MODULES
}

\author{
Lingling $\operatorname{Tan}^{1} \S$, Tiwei Zhao ${ }^{2}$ \\ ${ }^{1,2}$ School of Mathematics and Computer Science \\ Hubei University \\ Wuhan, 430062, P.R. CHINA
}

\begin{abstract}
Let $R$ be a ring, $n$ a fixed non-negative integer and $\mathcal{G I}_{n}\left(\mathcal{G F}_{n}\right.$, respectively) the class of all left $R$-modules of Gorenstein injective(Gorenstein flat, respectively) dimension at most $n$. A left $R$-module $M$ is called $n-G I-$ injective $\left(n-G F\right.$-projective, respectively) if $\operatorname{Ext}_{R}^{1}(N, M)=0\left(\operatorname{Ext}_{R}^{1}(M, N)=0\right.$, respectively) for any $N \in \mathcal{G I}_{n}\left(N \in \mathcal{G F}_{n}\right.$, respectively). A right $R$-module $M$ is called $n$-GI-flat if $\operatorname{Tor}_{1}^{R}(M, N)=0$ for any $N \in \mathcal{G} \mathcal{I}_{n}$. It is shown that a left $R$-module $M$ over any ring $R$ is $n$-GI-injective if and only if it is a kernel of a $\mathcal{G I}_{n}$-precover $f: A \rightarrow B$ with $A$ injective. If $R$ is an $n$-Gorenstein ring, then a finitely presented right $R$-module $M$ is $n$-GI-flat if and only if $M$ is a cokernel of a $\mathcal{G F}_{n}$-preenvelope $K \rightarrow F$ of a right $R$-module $K$ with $F$ flat. In addition, we present some applications about these classes of modules.
\end{abstract}

AMS Subject Classification: 16E30, 16E10

Key Words: $n$ - $G I$-injective, $n$ - $G F$-projective, $n$ - $G I$-flat, $\mathcal{G} \mathcal{I}_{n}$-precover, $\mathcal{G F}_{n^{-}}$ preenvelope

\section{Introduction}

Let $R$ be a ring. A left $R$-module $M$ is called $G I$-injective [5] if $\operatorname{Ext}_{R}^{1}(N, M)=0$ for any Gorenstein injective left $R$-module $N$, and $M$ is called strongly $G I$ -

Received: March 3, 2013

(c) 2013 Academic Publications, Ltd.

${ }^{\S}$ Correspondence author url: www.acadpubl.eu 
injective [5] if $\operatorname{Ext}_{R}^{i}(N, M)=0$ for any Gorenstein injective left $R$-module $N$ and any $i \geq 1$. A right $R$-module $M$ is called $G I$-flat [6] if $\operatorname{Tor}_{1}^{R}(M, N)=0$ for any Gorenstein injective left $R$-module $N$, and $M$ is called strongly $G I$ flat [6] if $\operatorname{Tor}_{i}^{R}(M, N)=0$ for any Gorenstein injective left $R$-module $N$ and any $i \geq 1$. GI-injective modules and $G I$-flat modules were discovered when studying Gorenstein injective precovers and Gorenstein flat preenvelopes, see to $[5,6]$ for details.

In basic homological algebra, the projective, injective and flat dimensions of modules play an important role. In the 2004's, the closely related Gorenstein projective, Gorenstein injective and Gorenstein flat dimensions were given and studied by H. Holm [8]. The Gorenstein injective dimension, $\operatorname{Gid}_{R}(M)$, of an $R$-module $M$ is defined by declaring that $\operatorname{Gid}_{R}(M) \leq n$ if and only if $M$ has a Gorenstein injective resolution of length $n$. Similarly, one define the Gorenstein projective dimension, $\operatorname{Gpd}_{R}(M)$, and Gorenstein flat dimension, $\operatorname{Gf}_{R}(M)$, of $M$, respectively.

In the 2007's, Mao and Ding in [9] introduced the notions of $n$-copure injective modules and $n$-copure flat modules for a fixed non-negative integer $n$. In the 2012's, Fu, Zhu and Ding in [3] introduced the notion of $n$-copure projective modules for a fixed non-negative integer $n$. Moreover, Duan and Ouyang (2011) in [1] and Gao (2012) in [4] introduced the notions of $n$-FI injective modules and $n-F I$ flat modules for a fixed non-negative integer $n$. Inspired by this, in this paper, we will introduce concepts of $n$ - $G I$-injective, $n$ $G F$-projective and $n$-GI-flat modules for a fixed non-negative integer $n$, which are generalizations of $[5,6]$.

In Section 2 of this paper, we introduce the concepts of $n$ - $G I$-injective, $n$ $G F$-projective and $n$-GI-flat modules for a fixed non-negative integer $n$ and show some of their properties. Let $R$ be a ring, $n$ a fixed non-negative integer and $\mathcal{G I}_{n}\left(\mathcal{G F}_{n}\right.$, respectively) the class of all left $R$-modules of Gorenstein injective(Gorenstein flat, respectively) dimension at most $n$. A left $R$-module $M$ is called $n$-GI-injective( $n$-GF-projective, respectively) if $\operatorname{Ext}_{R}^{1}(N, M)=0$ $\left(\operatorname{Ext}_{R}^{1}(M, N)=0\right.$, respectively) for any $N \in \mathcal{G I}_{n}\left(N \in \mathcal{G} \mathcal{F}_{n}\right.$, respectively). and a right $R$-module $M$ is called $n$-GI-flat if $\operatorname{Tor}_{1}^{R}(M, N)=0$ for any left $R$-module $N$ with $N \in \mathcal{G I}_{n}$. It is shown that a left $R$-module $M$ over any $\operatorname{ring} R$ is $n$-GIinjective if and only if it is a kernel of a $\mathcal{G I}_{n}$-precover $f: A \rightarrow B$ with $A$ injective and $M$ is $n$-GF-projective if and only if it is a cokernel of a $\mathcal{G F}_{n}$-preenvelope $f: A \rightarrow B$ with $B$ projective. It is also shown that a right $R$-module $M$ over any ring $R$ is $n$-GI-flat if and only if $M^{*}$ is $n$-GI-injective if and only if the functor $M \otimes_{R}$ - is exact with respect to each sequence $0 \rightarrow A \rightarrow B \rightarrow C \rightarrow 0$ of left $R$-modules with $C \in \mathcal{G I}_{n}$. If $R$ is an $n$-Gorenstein ring, then a finitely 
presented right $R$-module $M$ is $n$-GI-flat if and only if $M$ is a cokernel of a $\mathcal{G F}_{n}$-preenvelope $K \rightarrow F$ of a right $R$-module $K$ with $F$ flat.

In Section 3 of this paper, we present some applications. We show that $n$ - $G I$-injective modules coincide with strongly $G I$-injective modules over an $n$ Gorenstein ring. Let $R$ be a right noetherian ring, then every finitely generated $n$ - $G I$-flat right $R$-module is $n$-GF-projective. We also give some characterizations of commutative von Neumann regular.

In the following, we first recall some of notions and facts needed in this paper.

Let $R$ be a ring and $\mathcal{F}$ a class of $\mathrm{R}$-modules. By an $\mathcal{F}$-preenvelope of an R-module $M$ we mean a morphism $\varphi: M \rightarrow F$ where $F \in \mathcal{F}$ such that for any morphism $f: M \rightarrow F^{\prime}$ with $F^{\prime} \in \mathcal{F}$, there is a morphism $g: F \rightarrow F^{\prime}$ such that $g \circ \varphi=f$. If furthermore, when $F^{\prime}=F$ and $f=\varphi$ the only such $g$ are automorphisms of $F$, then $\varphi: M \rightarrow F$ is called an $\mathcal{F}$-envelope of $M$. Dually, we have the definition of $\mathcal{F}$-(pre)cover of an R-module. Note that $\mathcal{F}$-envelopes and $\mathcal{F}$-cover may not exist in general, but if they exist, they are unique up to isomorphism.

Given a class $\mathcal{C}$ of left $R$-modules, we will denote by $\mathcal{C}^{\perp}=\left\{M \mid \operatorname{Ext}_{R}^{1}(C, M)=\right.$ 0 for all $C \in \mathcal{C}\}$ the right orthogonal class of $\mathcal{C}$, and by ${ }^{\perp} \mathcal{C}=\left\{M \mid \operatorname{Ext}_{R}^{1}(M, C)=\right.$ 0 for all $C \in \mathcal{C}\}$ the left orthogonal class of $\mathcal{C}$. A pair $(\mathcal{F}, \mathcal{C})$ of classes of left $R$-modules is called a cotorsion theory if $\mathcal{F}^{\perp}=\mathcal{C}$ and ${ }^{\perp} \mathcal{C}=\mathcal{F}$. A cotorsion theory $(\mathcal{F}, \mathcal{C})$ is said to be perfect if every left $R$-module has a $\mathcal{C}$-envelope and an $\mathcal{F}$-cover. A cotorsion theory $(\mathcal{F}, \mathcal{C})$ is said to be hereditary if whenever $0 \longrightarrow L^{\prime} \rightarrow L \longrightarrow L^{\prime \prime} \rightarrow 0$ is exact with $L, L^{\prime \prime} \in \mathcal{F}$, then $L^{\prime}$ is also in $\mathcal{F}$, or equivalently, with $L, L^{\prime} \in \mathcal{C}$, then $L^{\prime \prime}$ is also in $\mathcal{C}$.

Throughout $R$ is an associative ring with identity and all modules are unitary. For an $R$-module $M, E(M)$ and $M^{*}$ stand for the injective envelope and character module of $M$, respectively. For unexplained concepts and notations, we refer the reader to $[2,11]$.

\section{Definitions and General Results}

We begin with the following.

Definition 1. Let $R$ be a ring, $n$ a fixed non-negative integer and $\mathcal{G I}_{n}\left(\mathcal{G F}_{n}\right.$, respectively) the class of all left $R$-modules of Gorenstein injective(Gorenstein flat, respectively) dimension at most $n$. A left $R$-module $M$ is called $n$-GIinjective if $\operatorname{Ext}_{R}^{1}(N, M)=0$ for any left $R$-module $N$ with $N \in \mathcal{G} \mathcal{I}_{n}$. A right $R$-module $M$ is called $n$-GI-flat if $\operatorname{Tor}_{1}^{R}(M, N)=0$ for any left $R$-module $N$ 
with $N \in \mathcal{G I}_{n}$. A left $R$-module $M$ is called $n$-GF-projective if $\operatorname{Ext}_{R}^{1}(M, N)=0$ for any left $R$-module $N$ with $N \in \mathcal{G F}_{n}$.

Remark 2. (1) By $[5,6], 0-G I$-injective modules were called $G I$-injective and $0-G I$-flat modules were called $G I$-flat. Moreover, if $m \geq n$, then $m$ - $G I$ injective modules, $m$ - $G I$ - flat modules and $m-G F$-projective modules are $n-G I$ injective, $n$ - $G I$-flat and $n$-GF-projective, respectively.

(2) A right $R$-module $M$ is $n$-GI-flat if and only if $M^{*}$ is $n$-GI-injective by the isomorphism: $\operatorname{Ext}_{R}^{1}\left(N, M^{*}\right) \cong \operatorname{Tor}_{1}^{R}(M, N)^{*}$ for any left $R$-module $N$.

(3) Let $I$ be any index set and $\left(M_{i}\right)_{i \in I}$ a family of left $R$-modules, then we have $\Pi_{i \in I} M_{i}$ is $n$-GI-injective if and only if $M_{i}$ is $n$-GI-injective for each $i$;

(4) Let $I$ be any index set and $\left(M_{i}\right)_{i \in I}$ a family of right $R$-modules, then we have $\oplus_{i \in I} M_{i}$ is $n$-GF-projective if and only if $M_{i}$ is $n-G F$-projective for each $i$;

(5) Let $I$ be any index set and $\left(M_{i}\right)_{i \in I}$ a family of right $R$-modules, then we have $\oplus_{i \in I} M_{i}$ is $n$-GI-flat if and only if $M_{i}$ is $n$-GI-flat for each $i$;

(6) The class of $n$-GI-injective left $R$-modules, the class of $n$ - $G F$-projective left $R$-modules and the class of $n$-GI-flat right $R$-modules are closed under extensions respectively.

Proposition 3. Let $R$ be a ring. Then

(1) If $\operatorname{Ext}_{R}^{i}(\widetilde{E}, M)=0$ for all $1 \leq i \leq n+1$ and all Gorenstein injective left $R$-modules $\widetilde{E}$, then every $k$-th cosyzygy of $M$ is $(n-k)$-GI-injective for any $0 \leq k \leq n$, in particular, $M$ is $n$-GI-injective.

(2) If $\operatorname{Ext}_{R}^{i}(M, \widetilde{F})=0$ for all $1 \leq i \leq n+1$ and all Gorenstein flat left $R$-modules $\widetilde{F}$, then every $k$-th syzygy of $M$ is $(n-k)-G F$-projective for any $0 \leq k \leq n$, in particular, $M$ is $n$-GF-projective;

(3) $\operatorname{If~}_{\operatorname{Tor}_{i}^{R}}(M, \widetilde{E})=0$ for all $1 \leq i \leq n+1$ and all Gorenstein injective left $R$-modules $\widetilde{E}$, then every $k$-th syzygy of $M$ is $(n-k)-G I$-flat for any $0 \leq k \leq n$, in particular, $M$ is $n$-GI-flat.

Proof. (1) Let $N \in \mathcal{G I}_{n-k}$. Consider the exact sequence

$$
0 \rightarrow M \rightarrow E^{0} \rightarrow E^{1} \rightarrow \cdots \rightarrow E^{k-1} \rightarrow V^{k} \rightarrow 0
$$

with $E^{i}$ injective. Let $V^{1}=\operatorname{Im}\left(M \rightarrow E^{0}\right), V^{i}=\operatorname{Im}\left(E^{i-2} \rightarrow E^{i-1}\right)$. Then $\operatorname{Ext}_{R}^{1}\left(N, V^{k}\right) \cong \operatorname{Ext}_{R}^{2}\left(N, V^{k-1}\right) \cong \cdots \cong \operatorname{Ext}_{R}^{k+1}(N, M)$. Since $N \in \mathcal{G I}_{n-k}$, we have an exact sequence

$$
0 \rightarrow N \rightarrow \widetilde{E}^{0} \rightarrow \widetilde{E}^{1} \rightarrow \cdots \rightarrow \widetilde{E}^{n-k} \rightarrow 0
$$


with $\widetilde{E}^{i}$ Gorenstein injective and hence

$$
\operatorname{Ext}_{R}^{k+1}(N, M) \cong \operatorname{Ext}_{R}^{n+1}\left(\widetilde{E}^{n-k}, M\right)=0
$$

by hypothesis. Thus, $\operatorname{Ext}_{R}^{1}\left(N, V^{k}\right)=0$ and so $V^{k}$ is $(n-k)$-GI-injective.

(2) Let $N \in \mathcal{G F}_{n-k}$. Consider the exact sequence

$$
0 \rightarrow L_{k} \rightarrow P_{k-1} \rightarrow \cdots \rightarrow P_{1} \rightarrow P_{0} \rightarrow M \rightarrow 0
$$

with $P_{i}$ projective. Let $L_{1}=\operatorname{Ker}\left(P_{0} \rightarrow M\right), L_{i}=\operatorname{Ker}\left(P_{i-1} \rightarrow P_{i-2}\right)$. Then $\operatorname{Ext}_{R}^{1}\left(L_{k}, N\right) \cong \operatorname{Ext}_{R}^{2}\left(L_{k-1}, N\right) \cong \cdots \cong \operatorname{Ext}_{R}^{k+1}(M, N)$. Since $N \in \mathcal{G F}_{n-k}$, we have an exact sequence

$$
0 \rightarrow \widetilde{F}_{n-k} \rightarrow \cdots \rightarrow \widetilde{F}_{1} \rightarrow \widetilde{F}_{0} \rightarrow N \rightarrow 0
$$

with $\widetilde{F}_{i}$ Gorenstein flat and hence $\operatorname{Ext}_{R}^{k+1}(M, N) \cong \operatorname{Ext}_{R}^{n+1}\left(M, \widetilde{F}_{n-k}\right)=0$ by hypothesis. Thus, $\operatorname{Ext}_{R}^{1}\left(L_{k}, N\right)=0$ and so $L_{k}$ is $(n-k)-G F$-projective.

(3) The proof is similar to that of (2), so we omit it here.

In the following, we give some characterizations of $n$ - $G I$-injective.

Proposition 4. Let $R$ be any ring and $M$ a left $R$-module. Then the following are equivalent:

(1) $M$ is $n$-GI-injective;

(2) For every exact sequence $0 \longrightarrow M \longrightarrow \widetilde{E} \stackrel{g}{\rightarrow} L \longrightarrow 0$ with $\widetilde{E} \in \mathcal{G I}_{n}$, $g: \widetilde{E} \rightarrow L$ is a $\mathcal{G} \mathcal{I}_{n}$-precover of $L$;

(3) $E(M) \rightarrow E(M) / M$ is a $\mathcal{G I}_{n}$-precover;

(4) $M$ is a kernel of a $\mathcal{G} \mathcal{I}_{n}$-precover $f: A \rightarrow B$ with $A$ injective;

(5) The functor $\operatorname{Hom}_{R}(-, M)$ is exact with respect to each exact sequence of left $R$-modules $0 \rightarrow A \rightarrow B \rightarrow C \longrightarrow 0$ with $C \in \mathcal{G I}_{n}$.

Proof. (1) $\Rightarrow(2)$ Let $M$ be $n$-GI-injective. For every exact sequence

$$
0 \rightarrow M \rightarrow \widetilde{E} \stackrel{g}{\rightarrow} L \rightarrow 0
$$

with $\widetilde{E} \in \mathcal{G} \mathcal{I}_{n}$, we have the following exact sequence

$$
\operatorname{Hom}_{R}(N, \widetilde{E}) \stackrel{g_{*}}{\rightarrow} \operatorname{Hom}_{R}(N, L) \rightarrow \operatorname{Ext}_{R}^{1}(N, M)
$$

with $N \in \mathcal{G I}_{n}$. By Definition $1, \operatorname{Ext}_{R}^{1}(N, M)=0$, and hence $\operatorname{Hom}_{R}(N, \widetilde{E}) \rightarrow$ $\operatorname{Hom}_{R}(N, L) \rightarrow 0$ is exact, that is, for any $h: N \rightarrow L$, there exists $h^{\prime}: N \rightarrow \widetilde{E}$ such that $g_{*}\left(h^{\prime}\right)=g h^{\prime}=h$. Hence $g: \widetilde{E} \rightarrow L$ is a $\mathcal{G} \mathcal{I}_{n}$-precover of $L$. 
$(2) \Rightarrow(3)$ First there exists a short exact sequence $0 \rightarrow M \rightarrow E(M) \rightarrow$ $E(M) / M \rightarrow 0$. Clearly, $E(M)$ is Gorenstein injective, thus $E(M) \in \mathcal{G I}_{n}$. By $(2), E(M) \rightarrow E(M) / M$ is a $\mathcal{G I}_{n}$-precover and hence (3) holds.

$(3) \Rightarrow(4)$ It is trivial.

$(4) \Rightarrow(1)$ Let $M$ be a kernel of a $\mathcal{G} \mathcal{I}_{n}$-precover $f: A \rightarrow B$ with $A$ injective. Then we have an exact sequence $0 \rightarrow M \rightarrow A \rightarrow B \rightarrow 0$. For any $N \in \mathcal{G I}_{n}$, the sequence

$$
\operatorname{Hom}_{R}(N, A) \rightarrow \operatorname{Hom}_{R}(N, B) \rightarrow \operatorname{Ext}_{R}^{1}(N, M) \rightarrow \operatorname{Ext}_{R}^{1}(N, A)=0
$$

is exact. But it is easy to verify that $\operatorname{Hom}_{R}(N, A) \rightarrow \operatorname{Hom}_{R}(N, B) \rightarrow 0$ is exact by (4). Thus $\operatorname{Ext}_{R}^{1}(N, M)=0$, and hence $M$ is $n$-GI-injective.

$(1) \Rightarrow(5)$ It is easy.

(5) $\Rightarrow(1)$ For any $N \in \mathcal{G I}_{n}$, there exists a short exact sequence

$$
0 \rightarrow K \rightarrow P \rightarrow N \rightarrow 0
$$

with $P$ projective. This induces an exact sequence

$$
\operatorname{Hom}_{R}(P, M) \rightarrow \operatorname{Hom}_{R}(K, M) \rightarrow \operatorname{Ext}_{R}^{1}(N, M) \rightarrow \operatorname{Ext}_{R}^{1}(P, M)=0 .
$$

But by (5), $\operatorname{Hom}_{R}(P, M) \rightarrow \operatorname{Hom}_{R}(K, M) \rightarrow 0$ is exact. Thus $\operatorname{Ext}_{R}^{1}(N, M)=$ 0 , and hence $M$ is $n$ - $G I$-injective.

Similarly, we have

Proposition 5. Let $R$ be any ring and $M$ a left $R$-module. Then the following are equivalent:

(1) $M$ is $n$-GF-projective;

(2) For every exact sequence $0 \rightarrow K \rightarrow \widetilde{F} \rightarrow M \rightarrow 0$ with $\widetilde{F} \in \mathcal{G F}_{n}$, $K \rightarrow \widetilde{F}$ is a $\mathcal{G} \mathcal{F}_{n}$-preenvelope of $K$;

(3) $M$ is a cokernel of a $\mathcal{G F}_{n}$-preenvelope $f: A \rightarrow B$ with $B$ projective;

(4) The functor $\operatorname{Hom}_{R}(M,-)$ is exact with respect to each exact sequence of left $R$-modules $0 \longrightarrow A \longrightarrow B \longrightarrow C \longrightarrow 0$ with $A \in \mathcal{G F}_{n}$.

Proposition 6. Let $R$ be any ring and $M$ a left $R$-module. Then the following are equivalent:

(1) $M$ is $n-G I$-flat;

(2) $M^{*}$ is $n-G I$-injective;

(3) $M \in^{\perp} \mathcal{C}$, where $\mathcal{C}=\left\{B^{*} \mid B \in \mathcal{G I}_{n}\right\}$;

(4) For every exact sequence $0 \rightarrow A \rightarrow B \rightarrow C \rightarrow 0$ of left $R$-modules with $C \in \mathcal{G I}_{n}$, the functor $M \otimes_{R}-$ preserves the exactness. 
Proof. (1) $\Leftrightarrow(2)$ It follows from Remark 2 .

$(1) \Leftrightarrow(3)$ It follows from the following standard isomorphisms: $\operatorname{Tor}_{1}^{R}(M, N)^{*}$ $=\operatorname{Ext}_{R}^{1}\left(M, N^{*}\right)$.

$(1) \Rightarrow(4)$ Let $M$ be $n$-GI-flat. Then for any exact sequence

$$
0 \rightarrow A \rightarrow B \rightarrow C \rightarrow 0
$$

of left $R$-modules with $C \in \mathcal{G} \mathcal{I}_{n}$, we have the following exact sequence

$$
0=\operatorname{Tor}_{1}^{R}(M, C) \rightarrow M \otimes_{R} A \rightarrow M \otimes_{R} B \rightarrow M \otimes_{R} C \rightarrow 0,
$$

and hence $M \otimes_{R}$ - is exact with respect to every exact sequence

$$
0 \rightarrow A \rightarrow B \rightarrow C \rightarrow 0
$$

of left $R$-modules with $C \in \mathcal{G I}_{n}$.

(4) $\Rightarrow(1)$ For any left $R$-module $N$ with $N \in \mathcal{G} \mathcal{I}_{n}$, there is an exact sequence of left $R$-modules $0 \rightarrow K \rightarrow P \rightarrow N \rightarrow 0$ with $P$ projective. This induces the following exact sequence

$$
\operatorname{Tor}_{1}^{R}(M, P) \rightarrow \operatorname{Tor}_{1}^{R}(M, N) \rightarrow M \otimes_{R} K \rightarrow M \otimes_{R} P \rightarrow M \otimes_{R} N \rightarrow 0
$$

with $\operatorname{Tor}_{1}^{R}(M, P)=0$.

On the other hand, $0 \rightarrow M \otimes_{R} K \rightarrow M \otimes_{R} P \rightarrow M \otimes_{R} N \rightarrow 0$ is exact by (4). Therefore $\operatorname{Tor}_{1}^{R}(M, N)=0$ for any left $R$-module $N$ with $N \in \mathcal{G I}_{n}$, and hence $M$ is $n$ - $G I$-flat.

Recall that a ring $R$ is called an $n$-Gorenstein ring if it is both left and right noetherian with self-injective dimension at most $n$ on both sides for some non-negative integer $n$.

Lemma 7. Let $R$ be an $n$-Gorenstein ring. If $M$ is a cokernel of a $\mathcal{G F}_{n^{-}}$ preenvelope $K \rightarrow F$ of right $R$-modules with $F$ flat, then $M$ is $n$ - $G I$-flat.

Proof. Assume that $M$ is a cokernel of a $\mathcal{G F}_{n}$-preenvelope $K \rightarrow F$ of right $R$-modules with $F$ flat. Let $L=\operatorname{Im}(K \rightarrow F)$, then we have an exact sequence $0 \rightarrow L \rightarrow F \rightarrow M \rightarrow 0$ and $L \rightarrow F$ is a $\mathcal{G F}_{n}$-preenvelope of $L$. Notes that for any $\widetilde{E} \in \mathcal{G I}_{n}$, by [2, Corollary 10.3.9], $\widetilde{E}^{*} \in \mathcal{G F}_{n}$ since $R$ is $n$-Gorenstein. Thus we obtain an exact sequence $\operatorname{Hom}_{R}\left(F, \widetilde{E}^{*}\right) \rightarrow \operatorname{Hom}_{R}\left(L, \widetilde{E}^{*}\right) \rightarrow 0$ by the definition of preenvelope. This gives rise to the exactness of sequence $\left(F \otimes_{R}\right.$ 
$\widetilde{E})^{*} \rightarrow\left(L \otimes_{R} \widetilde{E}\right)^{*} \rightarrow 0$, and hence $0 \rightarrow L \otimes_{R} \widetilde{E} \rightarrow F \otimes_{R} \widetilde{E}$ is exact. On the other hand, we have the following exact sequence

$$
0=\operatorname{Tor}_{1}^{R}(F, \widetilde{E}) \rightarrow \operatorname{Tor}_{1}^{R}(M, \widetilde{E}) \rightarrow L \otimes_{R} \widetilde{E} \rightarrow F \otimes_{R} \widetilde{E} .
$$

So $\operatorname{Tor}_{1}^{R}(M, \widetilde{E})=0$, and hence $M$ is $n$-GI-flat.

Theorem 8. Let $R$ be an $n$-Gorenstein ring. If $M$ is a finitely presented right $R$-module, then $M$ is $n$-GI-flat if and only if $M$ is a cokernel of a $\mathcal{G F}_{n^{-}}$ preenvelope $K \rightarrow F$ of right $R$-module with $F$ flat.

Proof. $\Leftarrow$ It follows from Lemma 7 .

$\Rightarrow$ Since $M$ is finitely presented, there is an exact sequence

$$
0 \rightarrow K \rightarrow P \rightarrow M \rightarrow 0
$$

with $P$ finitely generated projective and $K$ finitely generated. We claim that $K \rightarrow P$ is a $\mathcal{G F}_{n}$-preenvelope. Indeed, for any $F \in \mathcal{G F}_{n}$ we have $F^{*} \in \mathcal{G I}_{n}$ by [8, Proposition 3.11]. Thus $\operatorname{Tor}_{1}^{R}\left(M, F^{*}\right)=0$, and so we have the following commutative diagram:

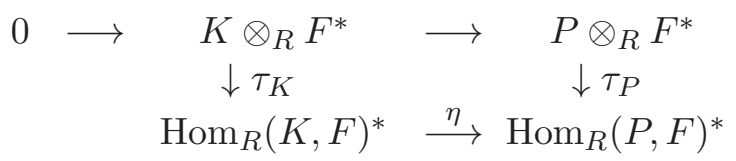

On the other hand, there exists an exact sequence $Q \rightarrow K \rightarrow 0$ with $Q$ finitely generated projective. Then we have the following commutative diagram:

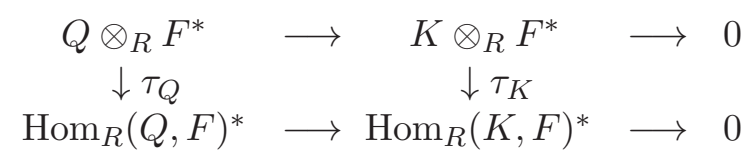

Note that $\tau_{Q}$ is an isomorphism by [11, Lemma 3.55], and hence $\tau_{K}$ is epimorphism. Since $\tau_{P}$ is an isomorphism, $\eta$ is a monomorphism. Thus $\operatorname{Hom}_{R}(P, F) \rightarrow$ $\operatorname{Hom}_{R}(K, F) \rightarrow 0$ is exact, i.e. $K \rightarrow P$ is a $\mathcal{G F}_{n}$-preenvelope.

Clearly, every injective(projective, flat, respectively) module is $n$ - $G I$-injective( $n$ - $G F$-projective, $n$ - $G I$-flat, respectively). However, $n$ - $G I$-injective $(n-G F$ projective, $n$-GI-flat, respectively) $R$-modules need not be injective(projective, flat, respectively) as shown by the following proposition. Recall that the Gorenstein injective dimension of a left $R$-module $M$ is defined to

l. $\operatorname{Gid}_{R}(M)=\inf \left\{n \mid\right.$ there is an exact sequence $0 \rightarrow M \rightarrow E_{0} \rightarrow E_{1}$

$\rightarrow \cdots \rightarrow E_{n} \rightarrow 0$ with $E_{i}$ Gorenstein injective for any $\left.0 \leq i \leq n\right\}$ 
Similarly, one define the Gorenstein projective dimension, $\operatorname{Gpd}_{R}(M)$, and Gorenstein flat dimension, $G f d_{R}(M)$, of $M$, respectively.

Proposition 9. Let $R$ be any ring.

(1) A left $R$-module $M$ is injective if and only if $M$ is $n$-GI-injective and l.Gid $R(M) \leq n+1$.

(2) A left $R$-module $M$ is projective if and only if $M$ is $n$-GF-projective and $r \cdot \operatorname{Gpd}_{R}(M) \leq n+1$.

(3) A right $R$-module $N$ is flat if and only if $N$ is $n$-GI-flat and $r \cdot G f d_{R}(N) \leq$ $n+1$.

Proof. (1) $\Rightarrow$ It is trivial.

$\Leftarrow$ Let $M$ be an $n$-GI-injective left $R$-module and $l \cdot G i d_{R}(M) \leq n+1$. Then there is an exact sequence $0 \rightarrow M \rightarrow E(M) \rightarrow E(M) / M \rightarrow 0$ with $E(M)$ injective. Since l.Gid $(M) \leq n+1$, l.Gid $_{R}(E(M) / M) \leq n$ by [10, Lemma 1.6]. Moreover, by Definition $1, \operatorname{Ext}_{R}^{1}(E(M) / M, M)=0$, so the exact sequence $0 \rightarrow M \rightarrow E(M) \rightarrow E(M) / M \rightarrow 0$ is split. Thus $M$ is injective as a direct summand of $E(M)$.

(2) It is similar to (1).

(3) $\Rightarrow$ It is trivial.

$\Leftarrow$ Let $N$ is $n$ - $G I$-flat and $r$. $G f d_{R}(N) \leq n+1$. We have $N^{*}$ is $n$-GI-injective

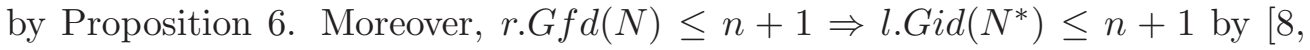
Proposition 3.11]. Thus $N^{*}$ is injective by (1). This implies $N$ is flat.

Proposition 10. Let $R$ be any ring, then the following are equivalent:

(1) Every right $R$-module is $n$ - $G I$-flat;

(2) Every finitely presented right $R$-module is $n$-GI-flat;

(3) Every left $R$-module $N$ with $N \in \mathcal{G I}_{n}$ is flat;

(4) Every cotorsion left $R$-module is $n$-GI-injective;

(5) Every pure injective left $R$-module is $n$-GI-injective.

Proof. (1) $\Rightarrow(2)$ It is trivial.

$(2) \Rightarrow(3)$ Let $N \in \mathcal{G} \mathcal{I}_{n}$. Then for any finitely presented right $R$-module $M$, by (2), it is $n$-GI-flat, and so $\operatorname{Tor}_{1}^{R}(M, N)=0$. This implies $N$ is flat.

$(3) \Rightarrow(4)$ Let $M$ be a cotorsion left $R$-module. For any $N \in \mathcal{G I}_{n}$, by (3) $N$ is flat, and so $\operatorname{Ext}_{R}^{1}(N, M)=0$ by the definition of cotorsion modules, which induces that $M$ is $n$ - $G I$-injective.

$(4) \Rightarrow(5)$ It follows from the fact that every pure injective module is cotorsion. 
$(5) \Rightarrow(1)$ Let $M$ be any right $R$-module, then $M^{*}$ is pure injective by $[2$, Proposition 5.3.7], and hence $M^{*}$ is $n$-GI-injective by (5). By Remark $2, M$ is n-GI-flat.

Proposition 11. Let $R$ be a ring. Then the following are equivalent:

(1) Every left $R$-module is $n$-GI-injective;

(2) Every left $R$-module $N$ with $N \in \mathcal{G I}_{n}$ is projective.

Proof. $(1) \Rightarrow(2)$ Let $N \in \mathcal{G I}_{n}$. For any left $R$-module $M$, by (1) $M$ is $n$-GI-injective, and so $\operatorname{Ext}_{R}^{1}(N, M)=0$, which implies $N$ is projective.

$(2) \Rightarrow(1)$ Let $M$ be any left $R$-module. For any $N \in \mathcal{G I}_{n}$, by (2) it is projective, and so $\operatorname{Ext}_{R}^{1}(N, M)=0$, which implies $M$ is $n$-GI-injective.

Similarly, we have

Proposition 12. Let $R$ be any ring. Then the following are equivalent:

(1) Every left $R$-module is $n$-GF-projective;

(2) Every left $R$-module $N$ with $N \in \mathcal{G F}_{n}$ is injective.

\section{Applications}

Recall from $[5,6]$ that a left $R$-module $M$ is called strongly $G I$-injective if $\operatorname{Ext}_{R}^{i}(N, M)=0$ for any Gorenstein injective left $R$-module $N$ and any $i \geq 1$ and a right $R$-module $\widetilde{M}$ is called strongly $G I$-flat if $\operatorname{Tor}_{i}^{R}(\widetilde{M}, N)=0$ for any Gorenstein injective left $R$-module $N$ and any $i \geq 1$. Now we call a left $R$ module $M$ strongly $G F$-projective if $\operatorname{Ext}_{R}^{i}(M, N)=0$ for any Gorenstein flat left $R$-module $N$ and any $i \geq 1$. Next we will say that $n$ - $G I$-injective modules coincide with strongly $G I$-injective modules over an $n$-Gorenstein ring.

Proposition 13. Let $R$ be an $n$-Gorenstein ring. Then a left $R$-module $M$ is $n$-GI-injective if and only if it is strongly GI-injective.

Proof. $\Rightarrow$ For any left $R$-module $N$, by [2, Theorem 12.3.1], the Gorenstein injective dimension of $N$ is at most $n$. On the other hand, consider the following exact sequence

$$
0 \rightarrow K_{i-1} \rightarrow P_{i-2} \rightarrow \cdots \rightarrow P_{1} \rightarrow P_{0} \rightarrow N \rightarrow 0
$$

with $P_{j}, 0 \leq j \leq i-2$ projective. By [2, Theorem 12.3.1] again, the Gorenstein injective dimension of $K_{i-1}$ is at most $n$ and so $\operatorname{Ext}_{R}^{i}(N, M) \cong \operatorname{Ext}_{R}^{1}\left(K_{i-1}, M\right)=$ 
0 . In particular, $\operatorname{Ext}_{R}^{i}(\widetilde{E}, M)=0$ for any Gorenstein injective module $\widetilde{E}$ and $i \geq 1$. Thus $M$ is strongly $G I$-injective.

$\Leftarrow$ Let $N$ be any left $R$-module. By [2, Theorem 12.3.1], l.Gid $\operatorname{Gi}_{R}(N) \leq n$. So there is an exact sequence

$$
0 \rightarrow N \rightarrow \widetilde{E}^{0} \rightarrow \widetilde{E}^{1} \longrightarrow \cdots \rightarrow \widetilde{E}^{m} \rightarrow 0
$$

with each $\widetilde{E}^{i}$ Gorenstein injective and $m \leq n$. Let $V^{0}=N, V^{i}=\operatorname{Im}\left(\widetilde{E}^{i-1} \rightarrow\right.$ $\left.\widetilde{E}^{i}\right)$ for $1 \leq i \leq m-1$ and $V^{m}=\widetilde{E}^{m}$. Then $0 \rightarrow V^{i} \rightarrow \widetilde{E}^{i} \rightarrow V^{i+1} \rightarrow 0,1 \leq$ $i \leq m-1$ are exact. For the first exact sequence $0 \rightarrow N \rightarrow \widetilde{E}^{0} \rightarrow V^{1} \rightarrow 0$, we have the following exact sequence

$$
\operatorname{Ext}_{R}^{1}\left(\widetilde{E}^{0}, M\right) \rightarrow \operatorname{Ext}_{R}^{1}(N, M) \rightarrow \operatorname{Ext}_{R}^{2}\left(V^{1}, M\right) \rightarrow \operatorname{Ext}_{R}^{2}\left(\widetilde{E}^{0}, M\right)
$$

By the hypothesis, $\operatorname{Ext}_{R}^{1}\left(\widetilde{E}^{0}, M\right)=0=\operatorname{Ext}_{R}^{2}\left(\widetilde{E}^{0}, M\right)$. $\quad$ So $\operatorname{Ext}_{R}^{1}(N, M) \cong$ $\operatorname{Ext}_{R}^{2}\left(V^{1}, M\right)$. By induction, we have

$$
\operatorname{Ext}_{R}^{1}(N, M) \cong \operatorname{Ext}_{R}^{2}\left(V^{1}, M\right) \cong \cdots \cong \operatorname{Ext}_{R}^{m+1}\left(\widetilde{E}^{m}, M\right)=0 .
$$

Thus $M$ is $n$ - $G I$-injective.

Proposition 14. Let $R$ be a right noetherian ring. Then every finitely generated $n$-GI-flat right $R$-module is $n$-GF-projective.

Proof. Let $M$ be finitely generated $n$-GI-flat. Since $R$ is right noetherian, by [11, Theorem 10.66], we have an isomorphisms:

$$
\operatorname{Ext}_{R}^{i}(M, N)^{*} \cong \operatorname{Tor}_{i}^{R}\left(M, N^{*}\right)
$$

for all $i \geq 1$. By [11, Example 3.64], every right noetherian ring is right coherent. On the other hand, we have $r \cdot \operatorname{Gid}_{R}\left(N^{*}\right)=r \cdot G f d_{R}(N)$ when $R$ is right coherent by [8, Proposition 3.11]. So the results hold.

By [7, Lemma 1.2.11], if $R$ is a right coherent ring and $M$ a finitely presented right $R$-module, then we also have an isomorphisms: $\operatorname{Ext}_{R}^{i}(M, N)^{*} \cong$ $\operatorname{Tor}_{i}^{R}\left(M, N^{*}\right)$ for all $i \geq 1$. So we have the following results.

Proposition 15. Let $R$ be a right coherent ring. Then every finitely presented $n$-GI-flat right $R$-module is $n$-GF-projective. 
Proposition 16. Let $R$ be a commutative noetherian ring and $M$ an $R$-module. Then

(1) $M$ is $n$-GI-injective if and only if $\operatorname{Hom}_{R}(P, M)$ is $n$-GI-injective for all projective $R$-modules $P$;

(2) $M$ is $n$-GI-flat if and only if $P \otimes_{R} M$ is $n$-GI-flat for all projective $R$-modules $P$.

Proof. $(1) \Leftarrow$ It is trivial by setting $P=R$.

$\Rightarrow$ Let $P$ be any projective $R$-module and $\widetilde{E} \in \mathcal{G} \mathcal{I}_{n}$. Then there exists an exact sequence $0 \rightarrow K \longrightarrow P^{\prime} \rightarrow \widetilde{E} \rightarrow 0$ with $P^{\prime}$ projective, which induces the exactness of the sequence

$$
0=\operatorname{Tor}_{1}^{R}(\widetilde{E}, P) \rightarrow K \otimes_{R} P \rightarrow P^{\prime} \otimes_{R} P \rightarrow \widetilde{E} \otimes_{R} P \rightarrow 0
$$

Note that $E \otimes_{R} P$ is Gorenstein injective for Gorenstein injective module $E$ and projective module $P$ by the proof of [4, Proposition 2.7]. Hence $\widetilde{E} \otimes_{R} P \in \mathcal{G} \mathcal{I}_{n}$. Then we have the following exact sequence

$$
\operatorname{Hom}_{R}\left(P^{\prime} \otimes_{R} P, M\right) \rightarrow \operatorname{Hom}_{R}\left(K \otimes_{R} P, M\right) \rightarrow \operatorname{Ext}_{R}^{1}\left(\widetilde{E} \otimes_{R} P, M\right)=0 .
$$

By [11, Theorem 2.75], we have the following exact sequence

$$
\operatorname{Hom}_{R}\left(P^{\prime}, \operatorname{Hom}_{R}(P, M)\right) \rightarrow \operatorname{Hom}_{R}\left(K, \operatorname{Hom}_{R}(P, M)\right) \rightarrow 0 \text {. }
$$

On the other hand, applying the functor $\operatorname{Hom}_{R}\left(-, \operatorname{Hom}_{R}(P, M)\right)$, we have the following exact sequence

$$
\begin{aligned}
& \operatorname{Hom}_{R}\left(P^{\prime}, \operatorname{Hom}_{R}(P, M)\right) \rightarrow \operatorname{Hom}_{R}\left(K, \operatorname{Hom}_{R}(P, M)\right) \\
& \rightarrow \operatorname{Ext}_{R}^{1}\left(\widetilde{E}, \operatorname{Hom}_{R}(P, M)\right) \rightarrow 0 .
\end{aligned}
$$

Therefore $\operatorname{Ext}_{R}^{1}\left(\widetilde{E}, \operatorname{Hom}_{R}(P, M)\right)=0$, and hence $\operatorname{Hom}_{R}(P, M)$ is $n$-GI-injective.

$(2) \Leftarrow$ It is trivial by setting $P=R$.

$\Rightarrow$ Let $P$ be any projective $R$-module. By Proposition 6 , it suffices to show that $\left(P \otimes_{R} M\right)^{*}$ is $n$-GI-injective. Indeed, $M$ is $n$-GI-flat $\Rightarrow M^{*}$ is $n$ - $G I$ injective $\Rightarrow \operatorname{Hom}_{R}\left(P, M^{*}\right)$ is $n$-GI-injective by $(1)$, and hence $\left(P \otimes_{R} M\right)^{*} \cong$ $\operatorname{Hom}_{R}\left(P, M^{*}\right)$ is $n$-GI-injective.

Corollary 17. Let $R$ be a commutative Artinian ring and $M$ an $R$-module. Then

(1) $M$ is $n$-GI-injective if and only if $\operatorname{Hom}_{R}(F, M)$ is $n$-GI-injective for all flat $R$-modules $F$; 
(2) $M$ is $n$-GI-flat if and only if $F \otimes_{R} M$ is $n$-GI-flat for all flat $R$-modules $F$.

Proof. It follows from the fact that a module is flat if and only if it is projective over a commutative Artinian ring.

In the following section, $\mathcal{G I F}_{n}$ stands for the class of all $n$-GI-flat right $R$-modules.

Proposition 18. Let $R$ be any ring. Consider the following:

(1) $\left(\mathcal{G I F}_{n}, \mathcal{G I F}_{n}^{\perp}\right)$ is a hereditary cotorsion theory;

(2) $\operatorname{Tor}_{2}^{R}(F, N)=0$ for any $F \in \mathcal{G I F}_{n}$ and $N \in \mathcal{G I}_{n}$;

(3) $\operatorname{Tor}_{j}^{R}(F, N)=0$ for any $F \in \mathcal{G I F}_{n}$ and $N \in \mathcal{G I}_{n}$ and $j \geq 1$.

(4) Every $n$-GI-flat right $R$-module is $m$-GI-flat for any $m \geq n$;

(5) Every $n$-GI-flat right $R$-module is $(n+1)$-GI-flat.

Then we have (1) $\Leftrightarrow(2) \Leftrightarrow(3)$. Moreover, if $R$ is a left noetherian ring with $\operatorname{Gid}\left({ }_{R} R\right) \leq n+1$, then (1) - (5) all are equivalent.

Proof. (1) $\Rightarrow(2)$ Let $F \in \mathcal{G I F}_{n}$, then there is an exact sequence

$$
0 \rightarrow K \rightarrow P \rightarrow F \rightarrow 0
$$

with $P$ projective. Since $\left(\mathcal{G I F}_{n}, \mathcal{G I F}_{n}^{\perp}\right)$ is hereditary, we have $K \in \mathcal{G I F}_{n}$. So we have the following exact sequence $0=\operatorname{Tor}_{2}^{R}(P, N) \rightarrow \operatorname{Tor}_{2}^{R}(F, N) \rightarrow$ $\operatorname{Tor}_{1}^{R}(K, N)=0$ with $N \in \mathcal{G} \mathcal{I}_{n}$, and hence $\operatorname{Tor}_{2}^{R}(F, N)=0$.

(2) $\Rightarrow(3)$ Let $F \in \mathcal{G I F}_{n}$ and $N \in \mathcal{G I}_{n}$. Then $\operatorname{Tor}_{1}^{R}(F, N)=0$ by Definition 1 , and so $\operatorname{Tor}_{j}^{R}(F, N)=0$ for any $j \geq 2$ by induction. Therefore (3) holds.

$(3) \Rightarrow(1)$ It is easy.

(3) $\Rightarrow(4)$ Let $F \in \mathcal{G I F}_{n}$ and $M \in \mathcal{G} \mathcal{I}_{m}$ with $m \geq n$. Then there is an exact sequence

$$
0 \rightarrow M \rightarrow \widetilde{E}^{0} \rightarrow \widetilde{E}^{1} \rightarrow \cdots \rightarrow \widetilde{E}^{m-n-1} \rightarrow V^{m-n} \rightarrow 0
$$

with each $\widetilde{E}^{i}$ Gorenstein injective. Note that $V^{m-n} \in \mathcal{G} \mathcal{I}_{n}$. Let $V^{1}=\operatorname{Im}(M \rightarrow$ $\left.\widetilde{E}^{0}\right), V^{i}=\operatorname{Im}\left(\widetilde{E}^{i-2} \rightarrow \widetilde{E}^{i-1}\right)$. By (3), we have

$$
\operatorname{Tor}_{1}^{R}(F, M) \cong \operatorname{Tor}_{2}^{R}\left(F, V^{1}\right) \cong \ldots \cong \operatorname{Tor}_{m-n+1}^{R}\left(F, V^{m-n}\right)=0 .
$$

Hence $F \in \mathcal{G I F}_{m}$.

$(4) \Rightarrow(5)$ It is trivial.

(5) $\Rightarrow(2)$ Let $F \in \mathcal{G I F}_{n}$. For any $N \in \mathcal{G I}_{n}$, there is an exact sequence $0 \rightarrow K \rightarrow P \rightarrow N \rightarrow 0$ with $P$ projective. By hypothesis, $P \in \mathcal{G I}_{n+1}$, and so $K \in \mathcal{G I}_{n+1}$ by [10, Lemma 1.6]. However, since $F \in \mathcal{G I F}_{n}$, by (5), we have $\operatorname{Tor}_{2}^{R}(F, N) \cong \operatorname{Tor}_{1}^{R}(F, K)=0$ for any $N \in \mathcal{G I}_{n}$, as desired. 
Proposition 19. Let $R$ be a commutative ring and $S$ a simple $R$-module. Then the following are equivalent:

(1) $S$ is $n$-GI-injective;

(2) $S$ is $n-G I$-flat;

(3) $S^{*}$ is $n-G I$-injective.

Proof. $(1) \Leftrightarrow(2)$ It follow from the proof of [9, Proposition 2.8.]

$(2) \Leftrightarrow(3)$ It is trivial by Proposition 6 .

Proposition 20. Let $R$ be a commutative ring. Then the following are equivalent:

(1) Every $R$-module is $n$-GI-flat;

(2) Every finitely presented $R$-module is $n$-GI-flat;

(3) Every $R$-module $N$ with $N \in \mathcal{G I}_{n}$ is flat;

(4) Every cotorsion $R$-module is $n$-GI-injective;

(5) Every pure injective $R$-module is $n$-GI-injective;

(6) $R$ is von Neumann regular.

Proof. $(1) \Leftrightarrow(2) \Leftrightarrow(3) \Leftrightarrow(4) \Leftrightarrow(5)$ are trivial by Proposition 10. It is enough to prove $(3) \Leftrightarrow(6)$.

$(3) \Rightarrow(6)$ By $(3)$, every Gorenstein injective module is flat, and so $R$ is von Neumann regular by [5, Proposition 4.2].

$(6) \Rightarrow(3)$ By $[11$, Theorem 4.9], a ring $R$ is von Neumann regular if and only if every $R$-module is flat. So (3) holds.

\section{References}

[1] L.L. Duan, B.Y. Ouyang, Relative FI-injective and FI-flat modules, Indian J. Pure Appl. Math., 42(6) (2011) 417-441.

[2] E.E. Enochs, O. M. G. Jenda, Relative Homological Algebra, Walter de Gruyter, Berlin-New York (2000).

[3] X.H. Fu, H.Y. Zhu, N.Q. Ding, On copure projective modules and copure projective dimensions, Comm. Algebra, 40 (2012), 343-359.

[4] Z.H. Gao, On n-FI-injective and n-FI-flat modules, Comm. Algebra, 40 (2012), 2757-2770.

[5] Z.H. Gao, On GI-injective modules, Comm. Algebra, 40 (2012), 3841-3858. 
[6] Z.H. Gao, On GI-flat modules and dimensions, J. Korean Math. Soc., 50(1) (2013), 203-218.

[7] R. Göbel, J. Trlifaj, Approximations and Endomorphism Algebra of Modules, Walter de Gruyter, Berlin-New York (2006).

[8] H. Holm, Gorenstein homological dimensions, J. Pure Appl. Algebra, 189 (2004), 167-193.

[9] L.X. Mao, N.Q. Ding, Relative copure injective and copure flat modules, J. Pure Appl. Algebra, 208 (2007), 635-646.

[10] N. Mahdou, M. Tamekkante, Strongly $n$-Gorenstein projective, injective and flat modules, arXiv: 0904.4013.

[11] J.J. Rotman, An introduction to homological algebra, 2nd ed. Springer, New York (2009).

[12] J. Xu, Flat covers of modules, Lecture Notes in Math., 1634, Spring-Verlag, Berlin (1996). 
